A detailed distribution of on-demand/daily analgesic/NSAID use is provided in the table below.

Table 1

\begin{tabular}{lllllc}
\hline Group of long-term users & \multicolumn{2}{c}{ NSAIDs } & Paracetamol & \begin{tabular}{c} 
Opioids \\
\cline { 2 - 5 }
\end{tabular} & $\begin{array}{c}\text { Combined } \\
\text { Non-selective Selective COX2 }\end{array}$ \\
\hline Pre-induction $(n=53)$ & $26 \%(17 \% / d)$ & $11 \%(4 \% / d)$ & $45 \%(8 \% / d)$ & $9 \%(6 \% / d)$ & $9 \%(2 \% / d)$ \\
Post-induction $(n=45)$ & $33 \%(16 \% / d)$ & $20 \%(7 \% / d)$ & $33 \%(4 \% / d)$ & $13 \%(7 \% / d)$ & $0 \%$ \\
\hline
\end{tabular}

Proportionally more patients were using NSAIDs in the post-induction group and paracetamol in the pre-induction group.

Mean DAS28CRP (BL-w104) in the pre-induction group was $3.11( \pm 0.70)$ and mean VAS pain $32.7( \pm 16.3)$. In this group $62.3 \%$ had sustained low disease activity while taking continuous analgesics/NSAIDs. Results in the post-induction group were comparable.

Analgesic/NSAID use was not significantly associated with mean (BL-w104) BMI, DAS28CRP or VAS pain.

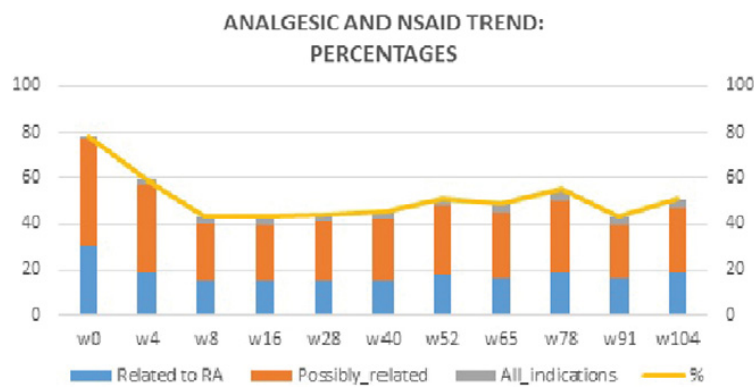

Conclusions: Analgesics/NSAID intake in early RA was high (90\%) and remarkably about $30 \%$ continued using these drugs, despite overall low disease activity. This high consumption might be explained by the ambiguous nature of arthritis-related pain and the lack of differentiation between nociceptive and non-nociceptive pain. Therefore pain management in early RA deserves more attention.

References:

[1] Verschueren, P. et al. ffectiveness of MTX with step-down glucocorticoid remission induction (COBRA Slim) vs other intensive treatment strategies for early RA in a treat-to-target approach: 1-year results of CareRA, a randomised pragmatic open-label superiority trial. Ann Rheum Dis.

Disclosure of Interest: None declared

DOI: 10.1136/annrheumdis-2017-eular.3399

\section{FRI0727 IMPACT OF MATERNAL SYSTEMIC AUTOIMMUNE RHEUMATIC DISEASES ON NEONATAL OUTCOMES: A POPULATION- LEVEL ANALYSIS}

S.O. Keeling, A. Savu, P. Kaul. Department of Medicine, University of Alberta, Edmonton, Canada

Background: The impact of systemic autoimmune rheumatic diseases (SARDS) on peripartum outcomes is not well described at a population level despite the potential for active disease in this period.

Objectives: We examined the association between SARDs and neonatal outcomes in a contemporary pregnancy cohort in the province of Alberta, Canada.

Methods: The patient population consisted of women giving birth between January 1, 2005 to December 31, 2014 ( $n=312,081)$. For women with multiple gestations during the period, one birth event was randomly selected. Women with SARDs included any of the following: systemic lupus erythematosus, systemic sclerosis, myositis and sjogren's syndrome, diagnoses based on the presence of International Classification of Disease version 9/10 codes in outpatient or inpatient records. Baseline characteristics, comorbidities, medication use (available for all births after January 1, 2009), and neonatal outcomes among women with and without SARDs were compared.

Results: Compared to women with no SARDs $(n=311,755)$, women with SARDs $(n=326,0.1 \%)$ were slightly older (SARDs 31.3 vs No SARDs 29.3 years $(p<0.01)$ ) but did not differ in terms of rural residence, ethnicity, median household income or nulliparity. However, rates of pre-term delivery, emergent cesearian section, induction, hypertensive disorders/eclampsia and mortality were higher among women with SARDs than those with no SARDs (Table 1). Offspring of women with SARDs had lower birth weights, were more likely small for gestational age (SGA), and had longer stays in neonatal ICU (Table 1). Among women with SARDs, prescription rates in the 270 days prior to delivery were highest for anti-malarials (Table 2). After multivariable adjustment, both NSAIDS use (OR (95\% CI): 5.24 $(1.57,17.52), p<0.01)$ and steroid use (OR (95\% Cl): $3.15(1.31,7.59), p<0.01)$ were significantly associated with a higher risk of preterm delivery.

Conclusions: Women with SARDs are at an increased risk of adverse outcomes during pregnancy. The association between corticosteroid and NSAID use and preterm delivery requires further investigation. Our findings suggest the need for
Table 1. Maternal and Neonatal Outcomes in SARDs vs No SARDs Mothers*

\begin{tabular}{lccc}
\hline Outcome & No SARDs \# (\%) & SARDs \# (\%) & P-value \\
\hline Pre-term Delivery & $22205(7.1)$ & $58(17.8)$ & $<0.01$ \\
C-section (Emergent) & $51132(16.4)$ & $82(25.2)$ & $<0.01$ \\
Hypertensive disorders & $20029(6.4)$ & $47(14.4)$ & $<0.01$ \\
Small for gestational age & $35047(11.2)$ & $68(20.9)$ & $<0.01$ \\
Congenital Anomaly & $5810(1.9)$ & $11(3.4)$ & 0.05 \\
Mean days in neonatal ICU & $0.8(5.0)$ & $2.3(9.6)$ & $<0.01$ \\
\hline *Sensitivity analysis utilizing primiparious SARDs vs NonSARDs women confirmed similar re-
\end{tabular}
sults.

Table 2. Unadjusted Effect of Medication Use During Pregnancy on Maternal and Neonatal Outcomes

\begin{tabular}{lccc}
\hline Outcome & Steroids & NSAIDS & Antimalarials \\
\hline \# (\%) SARDS patients on medication & $33(16.3)$ & $13(6.4)$ & $51(25.1)$ \\
SGA, OR $(95 \% \mathrm{Cl})$ & $2.06(0.91,4.67)$ & $1.08(0.29,4.12)$ & $1.54(0.74,3.20)$ \\
Gestational Diabetes, OR (95\% Cl) & $1.67(0.51,5.47)$ & $\mathrm{n} / \mathrm{a}$ & $0.91(0.28,2.93)$ \\
Preterm delivery, OR $(95 \% \mathrm{Cl})$ & $3.84(1.66,8.89)^{\star}$ & $4.96(1.55,15.85)^{\star}$ & $1.82(0.83,4.00)$ \\
C-section, OR $(95 \% \mathrm{Cl})$ & $0.88(0.41,1.90)$ & $0.98(0.31,3.11)$ & $0.57(0.29,1.13)$ \\
Hypertensive disorders of pregnancy, & & & \\
$\quad$ & $3.67(1.51,8.93)^{\star}$ & $0.50(0.06,4.03)$ & $1.23(0.51,2.99)$ \\
\hline OR $(95 \% \mathrm{Cl})$ & & &
\end{tabular}
${ }^{*} \mathrm{p}<0.05$

closer monitoring and coordinated care with obstetrics and perinatology in these high risk women.

Disclosure of Interest: None declared

DOI: 10.1136/annrheumdis-2017-eular.4561

\section{FRI0728 OSTEOARTHRITIS AND GOUT: REAL-WORLD EVIDENCE EVALUATING PATIENT CHARACTERISTICS, TREATMENT PATTERNS, AND HEALTHCARE UTILIZATION}

S. Krasnokutsky Samuels ${ }^{1}$, M. Pillinger ${ }^{1}$, S. Kabadi ${ }^{2}$, D.C. Taylor ${ }^{3}$, R. Morlock ${ }^{4}$ ${ }^{1}$ New York University, New York; ${ }^{2}$ AstraZeneca Pharmaceuticals, Gaithersburg;

${ }^{3}$ Ironwood Pharmaceuticals, Cambridge; ${ }^{4}$ YourCareChoice, Ann Arbor, United States

Background: Gout and osteoarthritis $(\mathrm{OA})$ are common in the United States, but little is known about potential associations of $O A$ and hyperuricemia/gout with clinical outcomes.

Objectives: This study examined variations in gout severity, management, and healthcare utilization among gout patients with and without $O A$.

Methods: Data were assessed from a survey of US physicians and patient chart audits. Participating physicians managed the care of $\geq 50$ patients with gout annually; chart audits were of their most recent 5 consecutive adult patients with confirmed gout. Gout severity was measured by physician global assessment, flares, organ/joint damage, and tophi. Treatment characteristics, presence of clinician-confirmed OA, and sociodemographic factors were identified. Descriptive and multivariate (stepwise logistic regression) statistics analyzed the differences among gout patients with and without clinician-confirmed comorbid OA, and assessed urate-lowering therapy (ULT) use and gout control.

Results: Overall, 1159 charts of gout patients were abstracted (230 w/ OA, $929 \mathrm{w} / 0 ; 81 \%$ male; $71 \%$ white); the proportion of patients aged $\geq 61$ was greater for those with gout and OA than those with gout but without OA $63 \%$ vs $32 \% ; P<0.001)$. Patients with gout and $O A$ had longer mean duration of gout (63 vs 41 months), were more likely to have tophi (44\% vs $19 \%$ ), joint damage (31\% vs $11 \%)$, and clinician-rated severe gout $(31 \%$ vs $12 \%)$ than those without $\mathrm{OA}$ (all $P<0.01$ ). Patients with gout and $\mathrm{OA}$ were also more likely to receive ULT $(89 \%$ vs $70 \% ; P<0.01)$, and among those receiving ULT, OA patients treated with allopurinol received a higher average daily dose (325 mg vs $296 \mathrm{mg} ; P=0.031)$. Gout patients with $O A$ were more likely to have additional comorbidities (cardiovascular disease, kidney disease, COPD, depression, diabetes, hyperlipidemia, hypertension, obesity, prostate problems [men]) and have chronic pain than those without $O A$ (all $P<0.05$ ). Gout patients with $O A$ reported more office visits (4.0 vs 3.5 ), were more likely to have an emergency department visit (17\% vs $9 \%$ ), and were more likely to require surgery for gout in the past 12 months ( $3 \%$ vs $0.3 \%$ ) (all $P<0.01$ ). In both groups, ULT use was associated with better gout control, but the specific factors predictive of ULT use and disease control varied between those with and without OA.

Conclusions: Gout patients with OA were more likely to have a greater impact on health system spending, with additional comorbidities and more severe gout than those without OA. These data suggest that gout patients with OA constitute a less healthy group in need of more careful monitoring and more aggressive gout management.

Acknowledgements: This study was sponsored by AstraZeneca.

Disclosure of Interest: S. Krasnokutsky Samuels: None declared, M. Pillinger: None declared, S. Kabadi Employee of: AstraZeneca, D. Taylor Employee of: Ironwood, R. Morlock Consultant for: AstraZeneca, Ironwood, Ardea Biosciences DOI: 10.1136/annrheumdis-2017-eular.5030 